\title{
Non-intercostal access for video-assisted thoracic surgery- analysis of technical advantages and disadvantages
}

\author{
Marcin Zieliński ${ }^{1}$, Pawel Gwozdz ${ }^{1}$, Michal Wilkojc ${ }^{1}$, Sylweriusz Kosinski ${ }^{2}$, Edward Fryzlewicz ${ }^{2}$, Tomasz \\ Nabialek $^{2}$, Juliusz Pankowski ${ }^{3}$, Robert Kwiatkowski ${ }^{4}$ \\ ${ }^{1}$ Department of the Thoracic Surgery, ${ }^{2}$ Department the Anaesthesiology and Intensive Care, ${ }^{3}$ Department of Pathology, Pulmonary Hospital, \\ Zakopane, Poland; ${ }^{4}$ Radiation Therapy, Katowice Oncology Center, Katowice, Poland \\ Contributions: (I) Conception and design: M Zieliński; (II) Administrative support: None; (III) Provision of study materials or patients: None; (IV) \\ Collection and assembly of data: M Zieliński, P Gwozdz, M Wilkojc, S Kosinski, E Fryzlewicz, T Nabialek, J Pankowski; (V) Data analysis and \\ interpretation: M Zieliński, R Kwiatkowski; (VI) Manuscript writing: All authors; (VII) Final approval of manuscript: All authors. \\ Correspondence to: Marcin Zieliński, MD, PhD. Department of Thoracic Surgery, Pulmonary Hospital, Ul. Gładkie 1, 34 500 Zakopane, Poland. \\ Email: marcinz@mp.pl.
}

\begin{abstract}
Background: Video-assisted thoracoscopic surgery (VATS) lobectomy has become an accepted method for the treatment of early-stage non-small-cell lung cancer (NSCLC). The standard VATS approach is an intercostal one which is often followed by postoperative pain due to injury of the intercostal nerve. The non-intercostal techniques of VATS include the subxiphoid, transcervical, transdiaphragmatic and transoral procedures.

Methods: The technical difficulty of operative management of the anatomical structures during VATS anatomical resection are compared for the intercostal, subxiphoid and transcervical approaches.

Results: Some operative steps have different range of difficulty, which are analyzed in detail.

Conclusions: The clearest advantages of the non-intercostal approaches include less postoperative pain and superradial bilateral mediastinal lymphadenectomy in case of the transcervical approach. However, the non-intercostal approaches are more technically demanding procedures, which therapeutic role has to be clarified in the future.
\end{abstract}

Keywords: Non-small-cell lung; intercostal nerves; video-assisted; pain; postoperative

Submitted Jun 14, 2018. Accepted for publication Sep 27, 2018.

doi: $10.21037 /$ jtd.2018.09.139

View this article at: http://dx.doi.org/10.21037/jtd.2018.09.139

\section{Introduction}

Video-assisted thoracoscopic surgery (VATS) lobectomy has become an accepted method for the treatment of early-stage non-small-cell lung cancer (NSCLC). The last edition of American College of Chest Physicians (ACCP) guidelines recommended that VATS was preferred over a thoracotomy for patients with clinical stage I NSCLC for anatomic pulmonary resection (1). This recommendation was based on the results of many non-randomized studies and subsequently supported by Bendixen et al., who prospectively compared postoperative pain and quality of life (QOL) in patients randomly assigned to the open anterolateral thoracotomy or 3-port VATS and discovered a significant advantage for VATS over thoracotomy (2). There are several variants of VATS lobectomy. The most accepted approach is an intercostal one with the use of one to several small incisions and without retraction of the ribs (3-6). In the recent years the uniportal approach described by Gonzales-Rivas has gained a world-wide interest (7). Furthermore, there are at least 3 variants of the intercostal uniportal VATS approach-anterior, posterior and axillary (7-10). Common disadvantage of all intercostal VATS technical variants is a substantial risk of development of a chronic postoperative pain and a decreased quality of life. The incidence of such pain after VATS was estimated for 
approximately $11-31.7 \%(11-14)$. Up to now, there was only one prospective study comparing uniportal vs multiportal VATS approach for anatomical pulmonary resections in regard to intensity of postoperative pain, which showed no difference between those techniques (15).

Possible advantages of the uniportal approach include avoidance of trocars and better geometry of performance of operations (16). There are possible disadvantages, which include quarreling of instruments, extra demands for thoracoscope-holding assistant and poorer ergonomy. Therefore, the problem of postoperative pain and the quality of life exists and for these reasons the other operative approaches are currently investigated.

Contemporarily, there are three alternative nonintercostal approaches including a subxiphoid, transcervical and transdiaphragmatic one. These techniques share one common feature of avoiding injury of the intercostal nerves and subsequent neuropathic pain.

\section{Subxiphoid approach}

This approach has been used for creation of a pericardial window, pericardiotomy and defibrillator implantation since the late seventies (17-19). More recently, the subxiphoid incision has been used for thymectomy, pulmonary metastasectomy and pulmonary lobectomies and segmentecties for lung cancer, including simultaneous bilateral resections (20-22). The subxiphoid approach should be evaluated separately for thymectomy and the other procedures of the anterior mediastinum and for pulmonary wedge resections and for anatomical pulmonary resection (lobectomies and segmentectomies).

\section{Thymectomy, mediastinal procedures}

There has been a fast-growing evidence for the virtues of the subxiphoid approach for thymectomy and the other anterior mediastinal procedures. Although no direct comparative studies for the intercostal VATS (unilateral or bilateral) and subxiphoid thymectomies has been ever undertaken, there are some undisputed advantages of the subxiphoid approach, including an access to both sides of the mediastinum with clear visibility of both phrenic nerves, which guarantees the completeness of an operation. The other advantage is a possibility to remove the content of the aorta-pulmonary window area, often containing ectopic thymic foci, which is not feasible in case of the right-sided VATS approach. The last and probably the most important advantage of the subxiphoid approach for thymectomy is a lack of chronic postoperative pain. There has been no single case of chronic postoperative pain in the group of 611 patients operated on at the Pulmonary Hospital in Zakopane with use of the subxiphoid approach (23). The incidence of the chronic postoperative pain in patients undergoing unilateral or bilateral VATS thymectomy has been never reported, nevertheless such pain is suffered by some patients.

In regard to the pulmonary metastasectomy it is possible to perform bilateral wedge pulmonary resection during one procedure.

\section{Anatomical pulmonary resections}

A subxiphoid approach has been used for anatomic pulmonary resection since 2014 (24).

Song et al. found the average pain scores significantly lower than those in the control group (standard intercostal uniportal VATS) (25). Disadvantages of the subxiphoid access include more difficulty in controlling bleeding with necessity to perform an additional VATS or open approach in case of major bleeding, less convenient access to the posterior aspect of the chest cavity and more difficult mediastinal lymphadenectomy. A specific, although rare complication of the subxiphoid approach is an postoperative abdominal wall hernia which occurred in 3/611 of our subxiphoid thymectomy group (23).

\section{Transcervical approach}

Historically, this approach was for the first time used by Sauerbruch, who performed thymectomy for myasthenia gravis in 1911 (26). Afterwards, transcervical thymectomy was reintroduced by Crile and later by Cooper et al. $(27,28)$. Our team used this approach for transcervical extended mediastinal lymphadenectomy and, subsequently for the right upper lobectomy and left upper lobectomy $(29,30)$. In 2016 we refined the technique of transcervical lobectomy and extended the operative indications to all pulmonary lobes with use of uniportal transcervical method (31). Tezel et al. has also described mediastinoscopic right upper lobectomy (32).

\section{Transdiaphragmatic approach}

Transdiaphragmatic approach which was described by Andrade $e t a l$. involves introduction of 4 laparoscopic ports including 2 intrathoracic ports inserted through diaphragmatic 
Table 1 The ease or difficulty of management of individual structures of the right pulmonary hilum by the intercostal VATS, subxiphoid and transcervical techniques

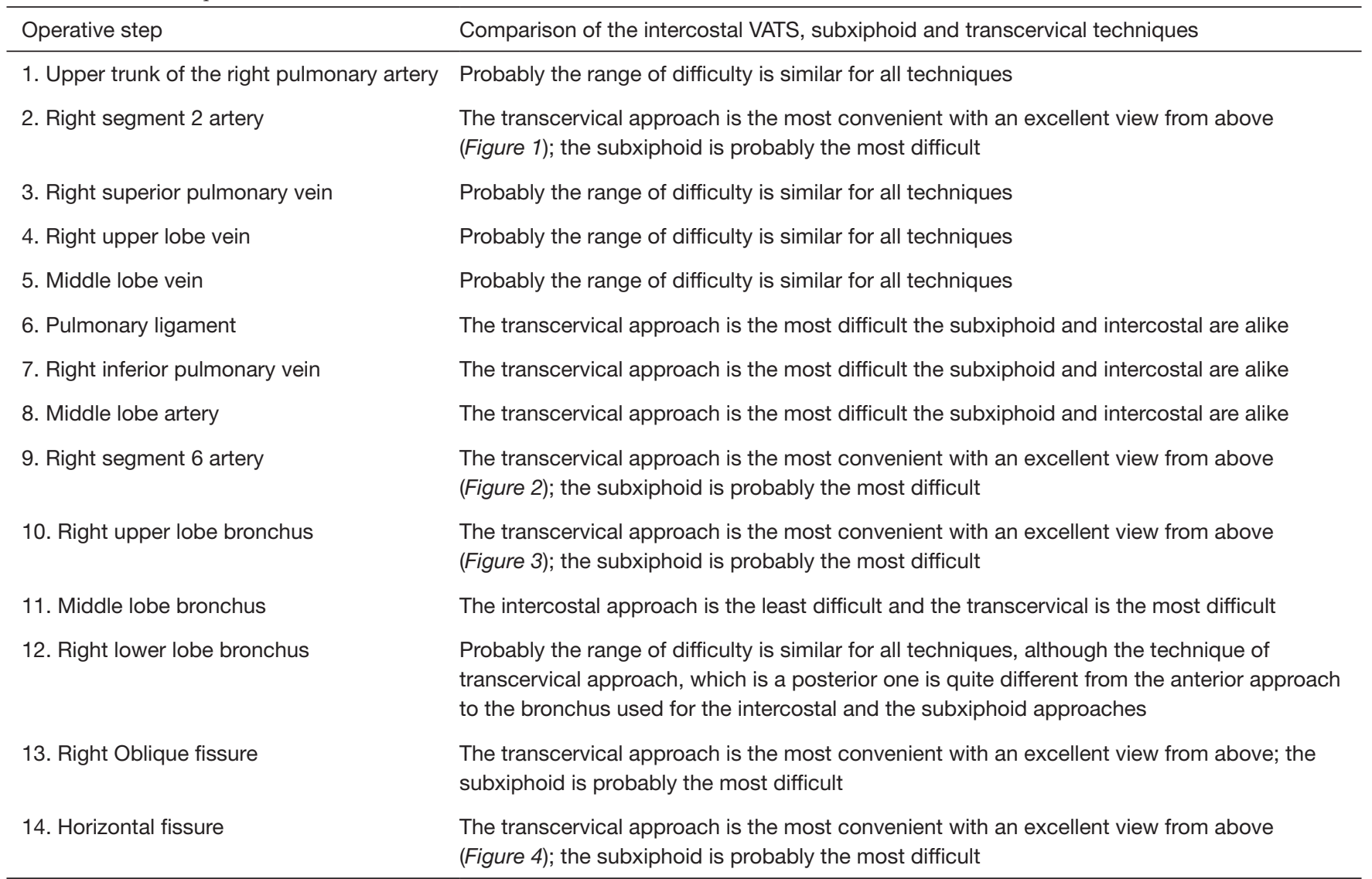

openings (33). An intrathoracic resection was performed in a standard VATS technique, a specimen was retrieved through the abdomen and the diaphragmatic openings which were finally closed. The main advantages of this technique was an elimination of the intercostal pain. The possible disadvantages included technical difficulties due to the long distance from the ports to the upper mediastinum, possible seeding of the abdominal cavity with cancer cells, possible intraabdominal formation of adhesions with a risk of subsequent ileus.

The aim of this article is to compare the basic technique of the intercostal VATS with two new non-intercostal approaches - the subxiphoid and the transcervical ones. At our institution we practice all these techniques, therefore we are going to try to present our own subjective opinion, which is only based on our clinical experience.

\section{Methods}

To compare the advantages and disadvantages of the intercostal, subxiphoid and transcervical approaches for anatomic pulmonary resection we decided to consider a pulmonary resection as a series of separate actions aimed to manage the pulmonary vessels, the bronchus and the parenchyma.

\section{Results}

The ease or difficulty of management of individual structures of the right and left pulmonary hilum and the mediastinal nodal stations by the intercostal VATS, subxiphoid and transcervical techniques are described in Tables 1-3.

We do not have any experience with pneumonectomy by the subxiphoid and transcervical approaches, therefore we cannot compare difficulty in management of the main bronchi and the main trunks of the pulmonary arteries

\section{Conclusions}

Introduction of the uniportal approach is probably a final 
Table 2 The ease or difficulty of management of individual structures of the left pulmonary hilum by the intercostal VATS, subxiphoid and transcervical techniques

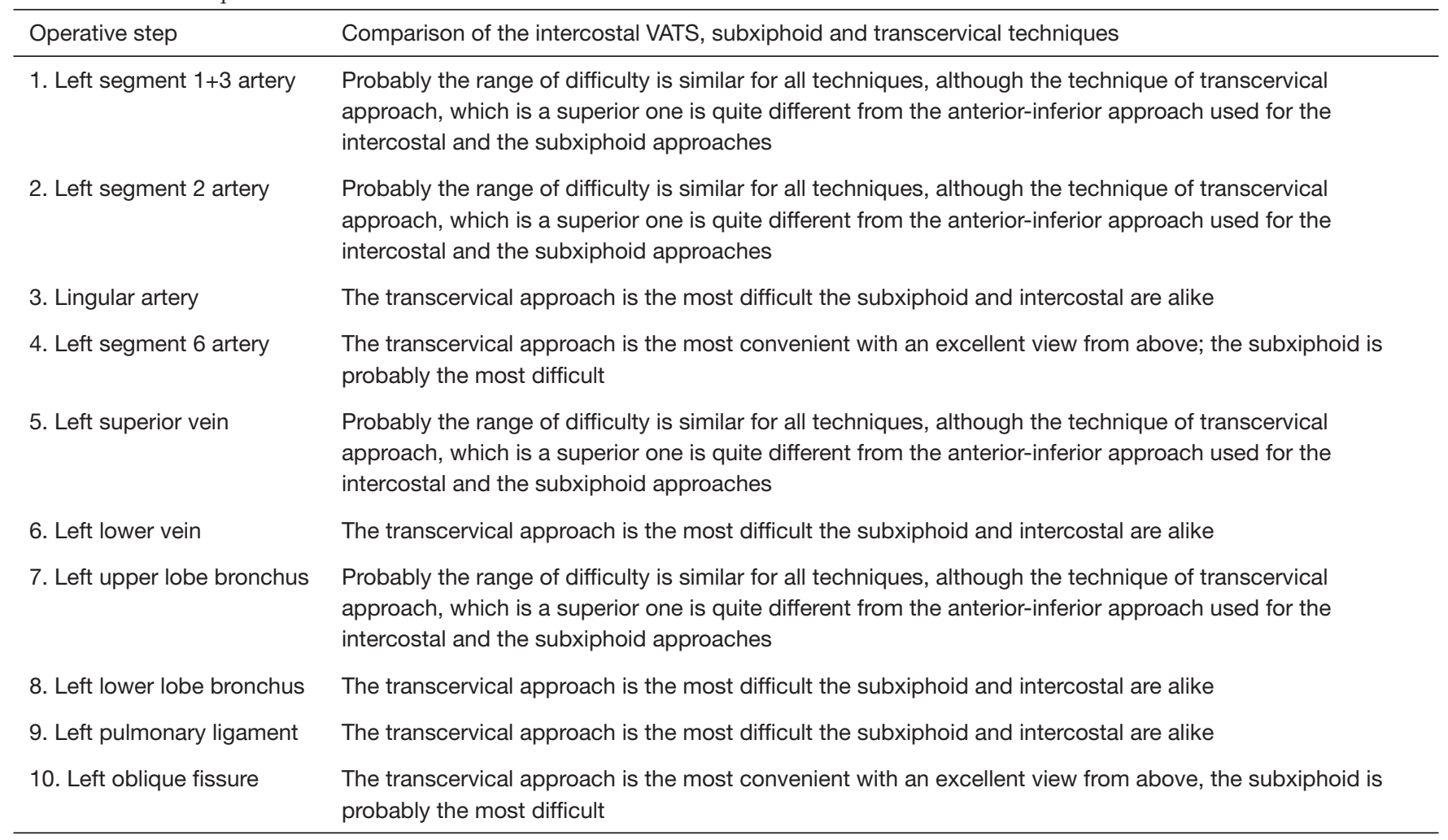

step in development of minimizing of the VATS approach with limitation to only one incision of 3-4 cm. However, there is a disadvantage of uniportal approach which is an intercostal incision, with possible risk of postoperative pain originating from injury of the intercostal nerve. To eliminate this risk the alternative uniportal approaches are currently studied, including subxiphoid and transcervical approaches, both of them avoiding the risk of chronic postoperative pain. Our experience in transcervical and subxiphoid operations assured us that a chronic postoperative pain never occurred in case of these approaches. Regarding the management of postoperative pain in patients undergoing transcervical lobectomies, no epidural or paravertebral analgesia is necessary due to the omission of any intercostal incisions. Our postoperative pain protocol includes treatment with nonsteroid anti-inflammatory drugs occasionally supplemented with narcotic drug injections, if necessary. In patients undergoing standard VATS lobectomy at our institution, we use epidural or paravertebral catheters. Additional advantages of subxiphoid and transcervical approaches is a creation of an opportunity to reach both pleural spaces and resect bilateral lesions during one procedure. An unique advantage of the transcervical approach is the possibility of performance of super-extended bilateral mediastinal lymphadenectomy by TEMLA preceding a pulmonary resection, with obtaining the results of the study of nodes intraoperatively (34). In case of the presence of the metastatic nodes it is possible to discontinue the procedure to avoid a resection in case of positive N2. Technical complexity and long duration of the procedures are disadvantages of the transcervical uniportal lobectomies. However, our experience with this kind of operations is still very limited, so with further progress and standardization of the details of surgical technique it is possible that the transcervical and subxiphoid approaches become easier and faster. Transcervical lobectomy is probably a more difficult technique than a uniportal lobectomy. Therefore, the other possible alternative to combine TEMLA and VATS lobectomy is to perform TEMLA and a typical uniportal VATS lobectomy independently during one anesthesia. It does not seem possible to perform transcervical lobectomy in non-intubated patients as has been proposed recently for the uniportal VATS and the subxiphoid VATS approaches $(35,36)$. Probably, more complex procedures 
Table 3 The ease or difficulty of management of the mediastinal nodal stations

Mediastinal nodal station
1. Station 1 mediastinal nodes
2. Station $2 \mathrm{R}$ mediastinal nodes
3. Station 3A mediastinal nodes
4. Station 3P mediastinal nodes
5. Station 4R mediastinal nodes
Comparison of the intercostal VATS, subxiphoid and transcervical techniques

Are not accessible for the intercostal and the subxiphoid approaches, easily accessible for the transcervical approach

Easily accessible for the transcervical approach, difficult for the intercostal and most difficult for the subxiphoid approach

Probably the range of difficulty is similar for all techniques, although the technique of transcervical approach, which is a superior one is quite different from the anterior-inferior approach used for the intercostal and the subxiphoid approaches

Rarely removed and difficult but the transcervical approach is the most convenient the subxiphoid and intercostal are alike

Most easily accessible for the transcervical approach and most difficult for the subxiphoid approach

6. Station 7 mediastinal nodes approached from The transcervical approach is the most convenient with an excellent view from above, the right side

the subxiphoid is probably the most difficult

7. Station 8 right mediastinal nodes

Probably the range of difficulty is similar for all techniques, although the technique of transcervical approach, which is a superior one is quite different from the anterior-inferior approach used for the intercostal and the subxiphoid approaches

\section{Station 9 right mediastinal nodes \\ 9. Station 5 mediastinal nodes}

The transcervical approach is the most difficult the subxiphoid and intercostal are alike

Probably the range of difficulty is similar for all techniques, although the technique of transcervical approach, which is a superior one is quite different from the anterior-inferior approach used for the intercostal and the subxiphoid approaches

10. Station 6 mediastinal nodes

Probably the range of difficulty is similar for all techniques, although the technique of transcervical approach, which is a superior one is quite different from the anterior-inferior approach used for the intercostal and the subxiphoid approaches

11. Station 7 mediastinal nodes approached The transcervical approach is the most convenient with an excellent view from above; the subxiphoid is probably the most difficult

from the left side

The transcervical approach is the most difficult, the subxiphoid and intercostal are alike

12. Station 8 left mediastinal nodes

13. Station 9 left mediastinal nodes

The transcervical approach is the most difficult, the subxiphoid and intercostal are alike

14. Station 10 right nodes

The transcervical approach is the most convenient the subxiphoid and intercostal are alike

15. Station 10 left nodes

The transcervical approach is the most convenient the subxiphoid and intercostal are alike

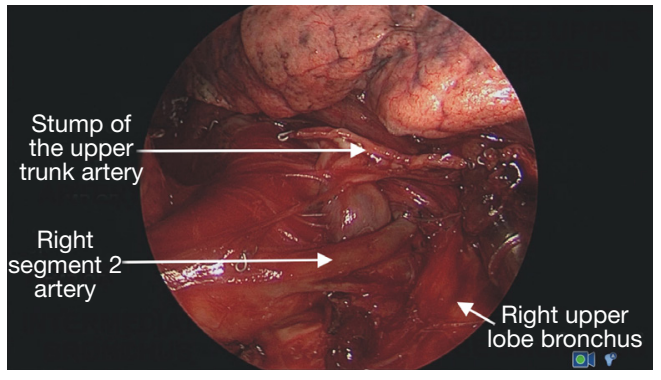

Figure 1 Right segment 2 artery-the view from the transcervical incision.

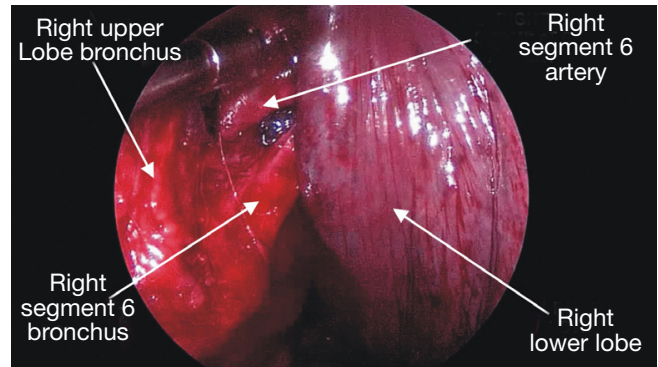

Figure 2 Right segment 6 artery-the view from the transcervical incision. 


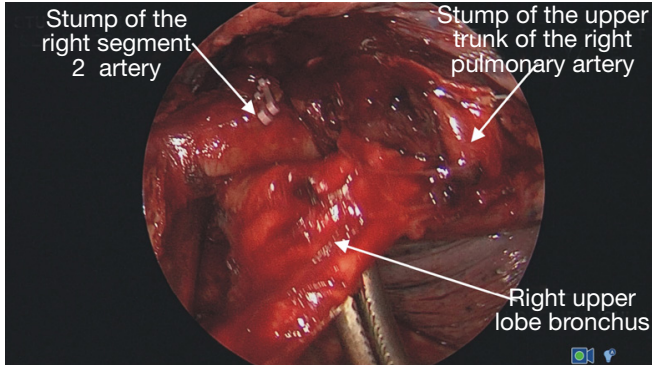

Figure 3 Right upper lobe bronchus-the view from the transcervical incision.



Figure 4 The horizontal fissure-the view from the transcervical incision.

as pneumonectomy, sleeve lobectomy, bronchovascular resections, carinal resection and the chest wall resection will not be possible with use of the transcervical approach, contrary to the intercostal uniportal approach (37). Currently, the transcervical approach is suitable only for relatively easy cases. However, it must be stressed that it was possible to perform lobectomy also in patients with total obliteration of the pleura space by adhesions.

Concluding, the clearest advantages of the nonintercostal approaches include less postoperative pain and superradial bilateral mediastinal lymphadenectomy in case of the transcervical approach. However, the non-intercostal approaches are more technically demanding procedures, which therapeutic role has to be clarified in the future.

\section{Acknowledgements}

None.

\section{Footnote}

Conflicts of Interest: The authors have no conflicts of interest to declare.

\section{References}

1. Howington J, Blum M, Chang A, et al. Treatment of stage I and II non-small cell lung cancer: Diagnosis and management of lung cancer, 3rd ed: American College of Chest Physicians evidence-based clinical practice guidelines. Chest 2013;143:e278S-313S.

2. Bendixen $M$, Jorgensen $\mathrm{O}$, Kronborg $\mathrm{C}$, et al. Postoperative pain and quality of life after lobectomy via video-assisted thoracoscopic surgery or anterolateral thoracotomy for early stage lung cancer: a randomised controlled trial. Lancet Oncol 2016;17:836-44.

3. McKenna R. Vats lobectomy with mediastinal lymph node sampling or dissection. Chest Surg Clin N Am 1995;5:223-32.

4. D'Amico TA, Niland J, Mamet R, et al. Efficacy of mediastinal lymph node dissection during lobectomy for lung cancer by thoracoscopy and thoracotomy. Ann Thorac Surg 2011;92:226-31; discussion 231-2.

5. Demmy TL, James TA, Swanson SJ, et al. Troubleshooting video-assisted thoracic surgery lobectomy. Ann Thorac Surg 2005;79:1744-52; discussion 1753.

6. Hansen HJ, Petersen RH, Christensen M. Videoassisted thoracoscopic surgery (VATS) lobectomy using a standardized anterior approach. Surg Endosc 2011;25:1263-9.

7. Gonzalez D, Paradela M, Garcia J, et al. Single-port videoassisted thoracoscopic lobectomy. Interact Cardiovasc Thorac Surg 2011;12:514-5.

8. Walker WS, Carnochan FM, Pugh GC. Thoracoscopic pulmonary lobectomy. Early operative experience and preliminary clinical results. J Thorac Cardiovasc Surg 1993;106:1111-7.

9. Stamenovic D, Bostanci K, Messerschmidt A. Posterior uniportal video-assisted thoracoscopic surgery for anatomical lung resections. J Thorac Dis 2017;9:5261-6.

10. Shen Y, Zhang Y, Sun J. Transaxillary uniportal video assisted thoracoscopic surgery for right upper lobectomy. J Thorac Dis 2018;10:E214-7.

11. Steegers MA, Snik DM, Verhagen AF, et al. Only half of the chronic pain after thoracic surgery shows a neuropathic component. J Pain 2008;9:955-61.

12. Koryllos A, Stoelben E. Video assisted thoracic surgery vs. thoracotomy regarding postoperative chronic pain. J Thorac Dis 2017;9:3498-500.

13. Bayman EO, Parekh KR, Keech J, et al. A prospective study of chronic pain after thoracic surgery. Anesthesiology 2017;126: 938-51. 
14. Wildgaard K, Ringsted T, Hansen H, et al. Persistent postsurgical pain after video-assisted thoracic surgery-an observational study. Acta Anaesthesiol Scand 2016;60:650-8.

15. Perna V, Carvajal A, Torrecilla J, et al. Uniportal videoassisted thoracoscopic lobectomy versus other videoassisted thoracoscopic lobectomy techniques: a randomized study. Eur J Cardiothorac Surg 2016;50:411-5.

16. Gonzalez-Rivas D, Sihoe A. Important Technical Details During Uniportal Video-Assisted Thoracoscopic Major Resections. Thorac Surg Clin 2017;27:357-72.

17. Arom KV, Franz J, Grover F, et al. Subxiphoid anterior mediastinal exploration. Ann Thorac Surg 1977;24:289-90.

18. Evans J, Gray L, Rayner A, et al. Principles for the management of penetrating cardiac wounds. Ann Surg 1979;189:777-84.

19. Watkins L, Mirowski M, Mower M, et al. Implantation of the automatic defibrillator: the subxiphoid approach. Ann Thorac Surg 1982;34:515-20.

20. Suda T, Ashikari S, Tochii S, et al. Single-incision subxiphoid approach for bilateral metastasectomy. Ann Thorac Surg. 2014;97:718-9.

21. Ito S, Tagawa T, Ide S, et al. Mediastinoscopic Extended thymectomy using a sternum lifting method. J Jpn Assoc Chest Surg 1997;11:181-5.

22. Hernandez-Arenas LA, Guido W, Jiang L. Learning curve and subxiphoid lung resections most common technical issues. J Vis Surg 2016;2:117.

23. Zieliński M, Gwozdz P, Solarczyk-Bombik K, et al. Video-assisted thoracic surgery thymectomy: subxiphoid approach. Mediastinum 2018;2:45.

24. Liu CC, Wang BY, Shih CS, et al. Subxiphoid singleincision thoracoscopic left upper lobectomy. J Thorac Cardiovasc Surg 2014;148:3250-1.

25. Song N, Zhao DP, Jiang L, et al. Subxiphoid uniportal video-assisted thoracoscopic surgery (VATS) for lobectomy: a report of 105 cases. J Thorac Dis 2016;8:S251-7.

Cite this article as: Zieliński M, Gwozdz P, Wilkojc M, Kosinski S, Fryzlewicz E, Nabialek T, Pankowski J, Kwiatkowski R. Non-intercostal access for video-assisted thoracic surgeryanalysis of technical advantages and disadvantages. J Thorac Dis 2018;10(Suppl 32):S3740-S3746. doi: 10.21037/jtd.2018.09.139
26. Keesey JC. A History of Treatments for Myasthenia Gravis. Seminars in Neurology 2004;24:5.

27. Kirschner PA. The history of surgery of the thymus gland. Chest Surg Clin N Am 2000;10:153-65.

28. Cooper JD, Al-Jilaihawa AN, Pearson FG, et al. An improved technique to facilitate transcervical thymectomy for myasthenia gravis. Ann Thorac Surg 1988;45:242-7.

29. Zieliński M. Technical pitfalls of transcervical extended mediastinal lymphadenectomy - how to avoid them and to manage intraooperative complications. Semin Thorac Cardiovasc Surg 2010;22:236-43.

30. Zielinski M, Pankowski J. Transcervical Right and Left Upper Pulmonary Lobectomies. In: Zielinski M, RamiPorta R (eds). Transcervical Approach in Thoracic Surgery. Springer 2014, p 159-64.

31. Zieliński M, Nabialek T, Pankowski J. Transcervical uniportal pulmonary lobectomy. JOVS pulmonary transcervical. J Vis Surg 2018;4:42.

32. Tezel C, Dogruyol T, Baysungur V, et al. The Most Minimally Invasive Lobectomy: Videomediastinoscopic Lobectomy. Surg Laparosc Endosc Percutan Tech 2016;26:e73-4.

33. Andrade RS, Diaz-Gutierrez I, Hutchins J, et al. Laparoscopic transdiaphragmatic chest surgery: Early experience. J Thorac Cardiovasc Surg 2018;155:1294-9.

34. Jakubiak M, Pankowski J, Obrochta A, et al. Fast cytological evaluation of lymphatic nodes obtained during transcervical extended mediastinal lymphadenectomy $\dagger$. Eur J Cardiothorac Surg 2013;43:297-301.

35. Jiang L, Liu J, Shao W, et al. Non-intubated subxiphoid uniportal video-assisted thoracoscopic thymectomy using glasses-free 3D vision. J Thorac Dis 2016;8:E1602-4.

36. Hwang J, Min TJ, Kim DJ, et al. Non-intubated single port thoracoscopic procedure under local anesthesia with sedation for a 5 -year-old girl. J Thorac Dis 2014;6:E148-51.

37. Gonzalez-Rivas D, Fieira E, Delgado M, et al. Uniportal video-assisted thoracoscopic sleeve lobectomy and other complex resections. J Thorac Dis 2014;6:S674-81. 